Einführung zum Thema

Pneumologe 2021 · 18:73-74

https://doi.org/10.1007/s10405-021-00380-7

Angenommen: 15. Januar 2021

(c) Springer Medizin Verlag $\mathrm{GmbH}$, ein Teil von Springer Nature 2021

Redaktion

M.A. Rose, Stuttgart

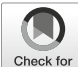

Markus A. Rose

Pädiatrische Pneumologie, Allergologie und CF Zentrum, Klinikum Stuttgart, Standort Mitte (Olgahospital), Stuttgart, Deutschland

\title{
Transition - von pädiatrischer in internistische lungenärztliche Versorgung
}

mit mannigfaltigen extrapulmonalen Organmanifestationen. Der interdisziplinäre Betreuungs- und Behandlungsbedarf dieser seltenen Erkrankungen zeigt die Bedeutung eines therapeutischen Netzwerkes. Eine bereitgestellte Checkliste ist ein schönes Beispiel eines Werkzeuges für eine strukturierte erfolgreiche Transition.

Die Mukoviszidose ist eine der klassischen Transitionskrankheiten mit jahrzehntelang bewährten Konzepten. F. Stehling et al. stellen dar, wie mit der kontinuierlich steigenden Lebenserwartung auch der quantitative und qualitative Bedarf an begleiteter Gesundheitsfürsorge zunehmen. So werden im Rahmen der historisch als „Kinderkrankheit“ geltenden CF (zystische Fibrose) komplexe Erwachsenen-spezifische Komorbiditäten wie CF-Diabetes mellitus oder CFHepatopathie beobachtet, die es adäquat zu verhindern und versorgen gilt.

Auch der pulmonalarterielle Hochdruck (PAH) bei Kindern hat sich mittlerweile von einer auch im Kindesalter fortschreitenden und lebensbedrohlichen Erkrankung zu einer Transitionskrankheit entwickelt. M. Gorenflo und V.C. Ziesenitz geben einen Überblick zu Therapiekonzepten, die hinsichtlich ihrer Wirksamkeit und Sicherheit bei Kindern bewertet werden. Aufgrund der unterschiedlichen Ätiologien wie PAH im Rahmen einer angeborenen Herzerkrankung können die Behandlungsstrategien bei Kindern im Vergleich zu Erwachsenen abweichen. Pharmakologische Herausforderungen in der pädiatrischen Altersgruppe wie therapeutisches DrugMonitoring oder der noch unerfüllte Be- darf an kontrollierten Studien mit PAHMedikamenten allein oder in Kombinationstherapie werden dargestellt. Das Beispiel der EMAH-Zusatzbezeichnung für Kardiologen (Betreuung angeborener Herzfehler im Erwachsenenalter) ist ein vorbildlicher Weg, die Transition zu institutionalisieren und professionalisieren.

\section{》) Je schwerer das Krankheits- bild, umso hilfreicher ist eine sorgfältige Transition}

Die neuromuskulären Erkrankungen mit chronisch respiratorischer Insuffizienz schließlich stellen eine interdisziplinäre Entität per se dar. M. Winterholler und $R$. Trollmann geben einen gerade für Lungenärzte faszinierenden Blick über den Tellerrand auf diese im Kindesalter auftretenden neuromuskulären Erkrankungen (NME), die entweder genetisch bedingt oder durch Autoimmunerkrankungen ausgelöst sind. Häufiger unter diesen insgesamt seltenen Erkrankungen sind die Muskeldystrophie Duchenne, die spinale Muskelatrophie und die hereditären Neuropathien, bei den Autoimmunerkrankungen die Myasthenia gravis, die Dermatomyositis sowie eine Reihe rheumatischer Erkrankungen. Je komplexer das Erkrankungsbild, je seltener die Erkrankung und je schwerer die Behinderung bei einem Adoleszenten ist, umso hilfreicher ist eine sorgfältige Transition. Dies gilt insbesondere auch für Patienten mit NME und Atemmuskelschwäche, bei denen ein Informationsverlust schnell $\mathrm{zu}$ vita- 
len Komplikationen führen kann. Eine Übergabe/Transition sollte frühzeitig geplant und kommuniziert werden.

Transition ist am effizientesten in etablierten Strukturen mit guten persönlichen Kontakten der Ärzte untereinander. Für diese Erkrankungen ist ein strukturierter Transitionsprozess wünschenswert, um die Jugendlichen auf den Wechsel der Versorgung vorzubereiten, den Informationsaustausch zwischen den Disziplinen sicherzustellen und im Sinne der Patienten eine erhöhte Morbidität durch Destabilisierung während eines unstrukturierten Wechsels zu verhindern. Die Phase der Transition fällt zudem in die Zeit der Pubertät, in der die Jugendlichen mehr Selbstständigkeit fordern und häufig deutlich risikobereiter sind mit der Konsequenz, dass die Therapieadhärenz sinkt, ohne dass vorab oder begleitend die Kompetenz für ein angemessenes Selbstmanagement der Erkrankung erworben wurde.

Wir wünschen unserer Leserschaft viel Freude an diesem Sonderheft!

Ihr

Prof. Dr. med. Dr. med. habil.

$$
17 \cdot \sqrt{2 a}
$$

Markus A. Rose

\section{Korrespondenzadresse}

Prof. Dr. med. Dr. med. habil. Markus A. Rose Pädiatrische Pneumologie, Allergologie und CF Zentrum, Klinikum Stuttgart, Standort Mitte (Olgahospital)

Kriegsbergstr. 62, 70174 Stuttgart, Deutschland m.rose@klinikum-stuttgart.de

Interessenkonflikt. M.A. Rose gibt an, dass kein Interessenkonflikt besteht.

\section{Unterschiede der Immunreaktion gegen SARS-CoV-2 bei milder und schwerer COVID-19-Krankheit}

Warum erkranken Menschen, die sich mit SARS-CoV-2 infiziert haben, unterschiedlich schwer an der davon ausgelösten Krankheit COVID-19?

Wissenschaftlerinnen und Wissenschaftler um Prof. Dr. Mascha Binder von der Universitätsmedizin Halle (Saale) haben dazu mehr als 14 Millionen RezeptorSequenzen von B- und T-Zellen, also Immunzellen, untersucht, die sie aus Blutproben von COVID-19-Patientinnen und -Patienten gewonnen haben. Die Ergebnisse hat die Gruppe im Fachmagazin „Immunity“ (CellPress) publiziert.

„Das Projekt zeigt Charakteristika von Immunantworten gegen Sars-CoV-2 bei milden und schweren Krankheitsverläufen auf. Die Unterschiede in den Immunantworten legen nahe, dass das Erreichen einer frühen schützenden Immunität oder auch das Verhältnis von schützenden und nichtschützenden Anteilen in der Immunantwort darüber entscheiden kann, ob eine schnelle Viruseliminierung und Abheilung gelingt und sich ein pathologischer Entzündungszustand und damit schwerer Erkrankungsverlauf vermeiden lässt. Unsere Daten geben somit einen grundlegenden Einblick in die erworbene Immunität gegen SARS-CoV-2", erklärt Mascha Binder, Professorin für Onkologie und Hämatologie sowie Direktorin der Universitätsklinik und Poliklinik für Innere Medizin IV der Universitätsmedizin Halle (Saale).

\section{Auffällige Laborwerte auch noch Wochen nach der Ausheilung}

Erstaunlich sei auch, dass man habe zeigen können, dass viele junge Patientinnen und Patienten mit milden Verläufen und schneller Erholung auch noch Wochen nach der Ausheilung auffällige Laborwerte zeigen. „Neben überschießend regenerierenden Immunzellen finden sich pathologische Muster in kardiovaskulären Risikofaktoren und in Interferonen, das sind bestimmte Botenstoffe, die in der viralen Abwehr eine Rolle spielen, aber auch mit Vermehrung von Bindegewebsfasern und Vernarbung in bestimmten Geweben wie der Lunge einhergehen können", erklärt die Wissenschaftlerin und Ärztin. Ob dies in dieser Patientengruppe mit einem erhöhten Risiko für Folgeerkrankungen assoziiert sei, müsse mit weiteren Langzeitbeobachtungsstudien geklärt werden.
Die Blutproben waren dabei sowohl von Menschen mit überstandener als auch von Menschen mit aktueller COVID-19-Erkrankung entnommen worden. Als Vergleichsgruppe diente eine altersangepasste Kohorte, die negativ auf COVID-19-Antikörper getestet wurde.

Doch nicht nur die Publikation steht der Wissenschaftswelt und Öffentlichkeit zur Verfügung, sondern auch die identifizierten Immunrezeptorsequenzen werden in einer öffentlichen Datenbank abgelegt. „Sie können damit von der wissenschaftlichen Gemeinschaft genutzt und zum Beispiel für diagnostische Anwendungen, aber auch zur Entwicklung einer passiven Immuntherapie mit neutralisierenden Antikörpern weiterentwickelt werden", so Binder.

Originalpublikation:

https://doi.org/10.1016/j.immuni.2020.06.024

\author{
Cornelia Fuhrmann, \\ Martin-Luther-Universität Halle- \\ Wittenberg
}

